\title{
Erratum to: Adenosine Monophosphate-Activated Protein Kinase Abates Hyperglycaemia-Induced Neuronal Injury in Experimental Models of Diabetic Neuropathy: Effects on Mitochondrial Biogenesis, Autophagy and Neuroinflammation
}

Veera Ganesh Yerra ${ }^{1}$ - Aparna Areti ${ }^{1}$. Ashutosh Kumar ${ }^{1}$

Published online: 8 October 2016

(C) Springer Science+Business Media New York 2016

\section{Erratum to: Mol Neurobiol}

DOI 10.1007/s12035-016-9824-3

In Fig 3 of the original version due to saturated fluorescence in mitosox staining the difference in the groups was not clearly evident, so we have re-performed these experiments and reestablished the Mitosox experiments and got a better representative images. Authors would like to include these corrected images as Fig. 3 in this erratum. This correction does not alter any of the conclusions claimed in the accepted article.
The authors do here by publish the new corrected Fig. 3 with updated Mitosox image panel.

The authors would also like to put forward a request to add Ms Aparna Areti as one of the co-authors as she has performed and reconfirmed the in vitro experimentation for images of mitosox submitted in this erratum.

On behalf of all the authors, I, Dr. Ashutosh Kumar testify the addition of Ms. Aparna Areti as a co-author.

The corrected list of authors and affiliations are hereby published.

The online version of the original article can be found at http://dx. doi.org/10.1007/s12035-016-9824-3.

Ashutosh Kumar

ashutosh.niperhyd@gov.in; ashutoshniper@gmail.com

Department of Pharmacology and Toxicology, National Institute of Pharmaceutical Education and Research (NIPER)-Hyderabad, Bala Nagar, India 


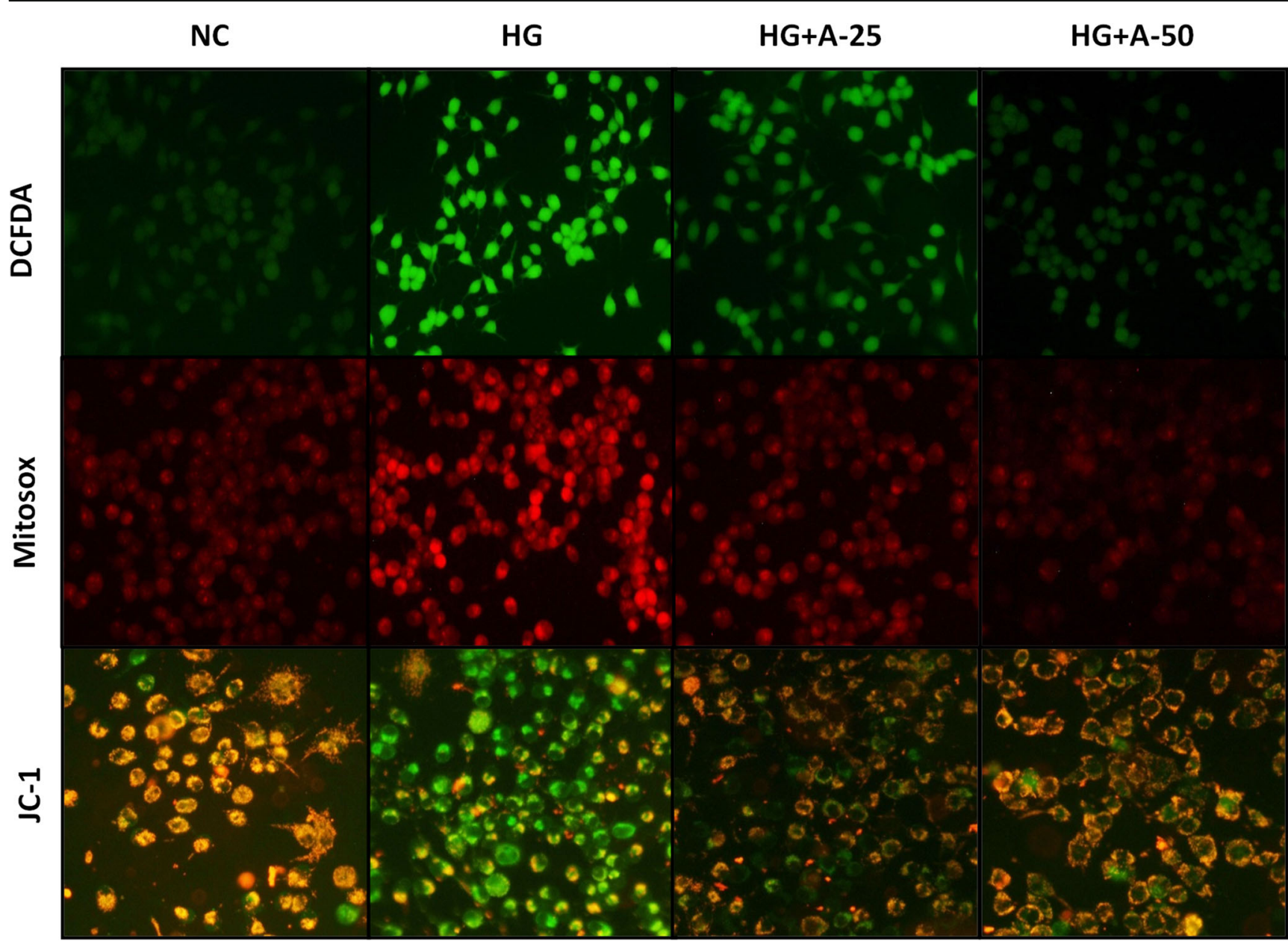

Fig. 3 Effect of high glucose exposure and AMPK activation on intracellular ROS levels, mitochondrial superoxide generation and mitochondrial membrane potential: representative DCF fluorescent images of ROS generation (first panel) in N2A cells exposed to high glucose and A769662. MitoSOX staining for the determination of mitochondrial superoxide generation (second panel) in N2A cells. JC-1 stained N2A cells for the determination of mitochondrial membrane potential. Orange fluorescence indicates the aggregated form of JC-1 and green colour is that of monomeric form. NC: normal N2A cells, $H G$ : high glucose (30 mM)-exposed N2A cells, $H G+A 25$ and $H G+A$ 50 are high glucose-insulted cells coincubated with A769662 at 25 and $50 \mu \mathrm{M}$, respectively 